\title{
Exact classical stochastic representations of the many-body quantum dynamics
}

\author{
E. A. Polyakov ${ }^{1}$, P. N. Vorontsov-Velyaminov ${ }^{1}$ \\ ${ }^{1}$ Saint Petersburg State University, \\ Dept. of molecular biophysics and polymer physics, Faculty of Physics, \\ 198504, Saint Petersburg, Russia \\ e.a.polyakov@gmail.com,voron.wgroup@gmail.com
}

PACS 05.30.Ch, 05.10.Gg, 02.70.-c, 02.70.Ss

DOI 10.17586/2220-8054-2015-6-4-501-512

In this work we investigate the exact classical stochastic representations of many-body quantum dynamics. We focus on the representations in which the quantum states and the observables are linearly mapped onto classical quasiprobability distributions and functions in a certain (abstract) phase space. We demonstrate that when such representations have regular mathematical properties, they are reduced to the expansions of the density operator over a certain overcomplete operator basis. Our conclusions are supported by the fact that all the stochastic representations currently known in the literature (quantum mechanics in generalized phase space and, as it recently has been shown by us, the stochastic wave-function methods) have the mathematical structure of the above-mentioned type. We illustrate our considerations by presenting the recently derived operator mappings for the stochastic wave-function method.

Keywords: quantum ensemble theory, quantum noise, stochastic equations.

Received: 20 June 2015

\section{Introduction}

At the theoretical department of Physical Faculty at Saint Petersburg State University, there is a scientific school with long-standing experience in various fields of stochastic systems, stochastic dynamics, and their applications to the physics of condensed matter. V.P. Romanov, to whose memory is devoted the current issue, contributed to this school by his works on stochastics aspects of light propagation in fluctuating nematic liquid crystals [1-3].

Over the past few decades, increasing efforts have been undertaken to devise and carry out the exact stochastic (Monte Carlo) simulations of many-body quantum dynamics. The field of research, which is the subject of such an activity, is truly interdisciplinary: quantum optics [4], utracold atoms in traps [5-7], quantum phase transitions [8], open quantum systems [9], and the measurement theory [10]. The motivation behind such studies range from purely practical (to provide exact data in order to verify a theory, or in order to interpret an experiment) to fundamental (to characterize the relationship between the classical and quantum computational complexity).

In order to devise a Monte Carlo simulation algorithm for quantum dynamics, we need to represent the quantum dynamics as an equivalent (with respect to average observable properties) probabilistic model. Such a model is called a stochastic representation. In this work, we focus only on classical diffusive stochastic representations of the reversible dynamics for quantum systems with pairwise interactions. Here, the adjective "classical" means that 
the quantum dynamics is mapped onto a classical stochastic process, rather than onto some quantum non-commutative extension of stochastic calculus [11].

Despite the fact that more than thirty years have passed since the fundamental work of Drummond et al. [4], where the first exact classical stochastic representation was introduced, this field of research still remains at an early stage of development. This is reflected in the fragmentedness of the literature on this subject: novel stochastic methods are constantly being invented [12-20], but based on entirely different and unrelated principles.

The classical stochastic representations of reversible quantum dynamics can be divided into two major groups: quantum dynamics in the generalized phase space, and stochastic wave-function methods. In our recent paper [21], we have demonstrated that the latter group of methods actually has the mathematical structure of the former, i. e. these two groups can be united into one common group.

In this work, we make an attempt to summarize various approaches in the literature [12-20] by defining the most general formulation for the classical stochastic representation of quantum dynamics. In Sec. 2, we define the notion of the classical stochastic representation. In such a representation, the mappings, which associate the classical quasiprobability distributions and functions with the quantum states and observables, appear as linear operators. In Sec. 3 we discuss (at the physical level of rigor) the general implications of the proposed definition of the classical stochastic representation. In particular, we demonstrate that, provided the mathematical properties of the representation are sufficiently regular, the representation reduces to an expansion of the density operator over a certain overcomplete operator basis. In Sec. 4, we illustrate our definitions by providing a concrete implementation of the classical stochastic representation: we present the recently derived [21] ordered operator correspondences for the stochastic wave-function method of Carusotto et al. [22].

\section{The classical stochastic representation}

Suppose that we are given a many-body quantum system with a Hamiltonian $\hat{H}$ :

$$
\widehat{H}=\sum_{r, s} h_{r s} \widehat{a}_{r}^{\dagger} \widehat{a}_{s}+\frac{1}{2} \sum_{r, s} V_{r s} \widehat{a}_{r}^{\dagger} \widehat{a}_{s}^{\dagger} \widehat{a}_{s} \widehat{a}_{r}
$$

containing a two-body interaction. Here, we assume that discretization of the continuous system was carried out [21], and the annihilation $\widehat{a}_{r}$ and creation $\widehat{a}_{s}^{\dagger}$ operators adhere to the bosonic commutation relations:

$$
\left[\widehat{a}_{r}, \widehat{a}_{s}^{\dagger}\right]=\delta_{r s}
$$

where $r$ and $s$ are the (multi-)indices of the discretization lattice sites [21]. The exact quantum evolution is governed by the full many-body quantum Liouville equation:

$$
\frac{\partial}{\partial t} \hat{\rho}=\frac{1}{i \hbar}[\widehat{H}, \widehat{\rho}(t)]
$$

where the density operator $\hat{\rho}(t)$ determines the state of the system at time $t$.

Summarizing various classical stochastic representations which are presented in the literature [12-20, 23, 24], we arrive at a general formulation for what we call here the "classical stochastic representation". We select a certain convex set $P_{+}$of physical density operators which are characteristic to our system. That is, if we take an arbitrary density operator $\hat{\rho}_{0} \in P_{+}$as the initial condition for Eq. (3) at $t=0$, then, at any later time $t$, the solution $\hat{\rho}(t)$ of Eq. (3) still belongs to the set $P_{+}$.

In a classical stochastic representation of quantum dynamics, the quantum state should be represented by a quasiprobability distribution in a certain (abstract) space $L$. The 
prefix 'quasi' means that such distributions do not correspond to any physical probabilities, and such distributions do not even satisfy the Kolmogorov axioms, since the elements $\lambda$ of the space $L$ cannot be interpreted as elementary events (usually, the Dirac distributions corresponding to the elements $\lambda$ do not correspond to any physical state). The space $L$ is usually called the generalized phase space [23-26], due to its intrinsic analogy to the phase space in the deformation quantization [27]. We suppose that the stochastic representation provides us with a methodology for how to assign a positive quasiprobability distribution $\mathcal{P}(\lambda)$ to any $\hat{\rho} \in P_{+}$. Such a procedure is given by the (possibly nonlinear) mapping $C_{\rho}$ :

$$
C_{\rho}[\hat{\rho}]=\mathcal{P}(\lambda) \text {, where } \mathcal{P}(\lambda) \geqslant 0 \text { for every } \lambda \in L .
$$

In a classical stochastic representation, the average value for the observable $\langle\hat{o}\rangle$ should be represented by a classical expectation over the quasiprobability. Therefore, we need a second mapping, $C_{o}$ :

$$
C_{o} \hat{o}=\mathcal{O}(\lambda)
$$

which defines how the quantum observables in our stochastic representation are delineated by classical functions $\mathcal{O}(\lambda)$ in the space $L$. Then, we have the expression for the expected value $\langle\hat{o}\rangle$ :

$$
\langle\hat{o}\rangle=\operatorname{Tr} \hat{\rho} \hat{\rho}=\int d \lambda \mathcal{O}(\lambda) \mathcal{P}(\lambda),
$$

which is required to be valid for every classical representation of the observable $\mathcal{O}(\lambda)$ and for every quasiprobability $\mathcal{P}(\lambda)$ from the image $\operatorname{im} C_{\rho}$. We assume that the mapping $C_{o}$ is a linear operator.

We are not only interested in the expected values of the observables, but also in their evolution over time. We want the time evolution in our representation to be represented in such a way, that it could be simulated by Monte Carlo methods. Thus, we need a third mapping, $C_{s}$ :

$$
C_{s}\{[\widehat{H}, \cdot] / i \hbar\}=\mathcal{H}
$$

This defines how the quantum Liouville superoperator $[\widehat{H}, \cdot] / i \hbar$ is being represented by such integro-differential operator $\mathcal{H}: D \rightarrow D$, that the quantum Liouville equation (3) is now represented by the differential Chapman-Kolmogorov master equation:

$$
\frac{\partial}{\partial t} \mathcal{P}=\mathcal{H} \mathcal{P}
$$

For example, if we are interested in a diffusive stochastic representation, we may look for such a stochastic representation which possesses such a mapping $C_{s}$ that $\mathcal{H}$ is a Fokker-Planck operator (in the Ito form [28]):

$$
\mathcal{H}=-\sum_{i} \frac{\partial}{\partial \lambda_{i}} \mathcal{A}_{i}(\lambda)+\frac{1}{2} \sum_{i, j} \frac{\partial^{2}}{\partial \lambda_{i} \partial \lambda_{j}} \mathcal{D}(\lambda),
$$

where $\lambda_{i}$ is a component of the vector $\lambda$ (if the space $L$ is multidimensional). In this case, we can simulate the quantum dynamics by numerically integrating the stochastic differential equation

$$
d \lambda_{i}=\mathcal{A}_{i}(\lambda) d t+\sum_{j} \mathcal{B}_{i j}(\lambda) d W_{j}
$$

where the Wiener increments have the standard statistical properties, $\mathrm{E}\left[d W_{i}\right]=0$ and $\mathrm{E}\left[d W_{i} d W_{j}\right]=\delta_{i j} d t ; \mathcal{D}=\mathcal{B B}^{T}[28]$. We assume that the mapping $C_{s}$ is a linear operator. 
The practical Monte Carlo simulation involves the following stages. First, we represent the initial condition $\hat{\rho}(t=0)$ as a positive quasiprobability distribution $\mathcal{P}(\lambda, t=0)$ using the mapping $C_{\rho}$. Then, we sample the initial conditions $\lambda(t=0)$ for the stochastic process (10) from the initial probability $\mathcal{P}(\lambda, t=0)$ using the importance sampling method [29]. Each sampled initial condition $\lambda(t=0)$ is propagated in time by the numerical integration of the stochastic differential equation (10). The average value of the observable $\langle\hat{o}\rangle(t)$ at a time $t$ is evaluated as the classical expectation $\langle\hat{o}\rangle(t)=\mathrm{E}[\mathcal{O}(\lambda(t))]$ over an ensemble of trajectories $\lambda(t)$.

\section{Some properties of the stochastic representations}

\subsection{The mapping for observables $C_{o}$}

Our classical stochastic model, as defined in the previous section by the tuple $\left(P_{+}, L\right.$, $C_{\rho}, C_{o}, C_{s}$ ), should bear a physical meaning. Let us examine what consequences can be drawn from this statement. The state of a physical system is completely defined by its observable properties. In the quantum case, the latter statement is true, since the density operator $\hat{\rho}$ is completely defined by all the moments of the canonically conjugate variables [30-32]. Therefore, the most important ingredient of our otherwise completely abstract model is the mapping $C_{o}$ for the observables. Let us write the trace formula (6) as a dot product:

$$
\langle\hat{o}\rangle=\operatorname{Tr} \hat{\rho} \hat{\rho}=\left\langle\mathcal{O}^{*}, \mathcal{P}\right\rangle_{S_{+}} .
$$

Suppose that all the observable operators $\hat{o}$ belong to some operator space $O_{+}$, and all their possible classical representaions $\mathcal{O}$ belong to some space $S_{+}$. Then, the mapping $C_{o}$ is acting between the spaces $O_{+}$and $S_{+}$,

$$
C_{o}: O_{+} \rightarrow S_{+}
$$

Suppose that $S_{+}$is chosen to be the minimal possible space, i.e. $S_{+}=\operatorname{im} C_{o}$. Note that the spaces $O_{+}$and $S_{+}$are real. Since the physical consistency requires that different $\hat{o}$ should be mapped onto different $\mathcal{O}$, we conclude that $C_{o}$ is a bijection. Now, since the average values of the observables are computed according to the dot-product formula (11), we see that one can actually introduce a space of quasiprobabilities $D$, for which the dot product (11) is well-defined for any $\mathcal{O} \in S_{+}$. We require that the space $D$ is such that one can introduce a dot-product $\langle\cdot, \cdot\rangle_{D}$ in it. Further, we call $D$ "the space of admissible quasiprobability distributions". From Eq. (11), it is seen that the space $D$ is split into two subspaces:

$$
D=R \oplus Z,
$$

where the subspace $Z$ consists of all such quasiprobabilities $\mathcal{Z}(\lambda)$ which are orthogonal to every $\mathcal{O}^{*}(\lambda) \in S_{+}^{*}:\left\langle\mathcal{O}^{*}, \mathcal{Z}\right\rangle_{S_{+}}=0$. The space $R$ is the orthogonal complement to $Z$ in the space $D$ (in the sense of the dot-product $\langle\cdot, \cdot\rangle_{D}$ ). If we add any element $\mathcal{Z} \in Z$ to the quasiprobability $\mathcal{P}$, then the observable properties of the state $\mathcal{P}+\mathcal{Z}$ are the same as those of $\mathcal{P}$. Therefore, if our model is physically consistent, the quasiprobabilities $\mathcal{P}$ and $\mathcal{P}+\mathcal{Z}$ should correspond to the same density operator $\hat{\rho}$. Hence, if the space $\mathcal{Z}$ is not trivial, it contributes to the non-uniqueness of the quasiprobability representation. The opposite is also true: every element $\mathcal{Z}$ such that it can be added to any $\mathcal{P}$ without changing the corresponding physical state $\hat{\rho}$, should belong to $Z$. We call the space $R$ the "minimal representation space", because (i) for every density operator $\hat{\rho}$, there is a unique quasiprobability $\mathcal{R} \in R$ such that it has the same observable properties as $\hat{\rho}$, and (ii) the quasiprobabiliy representation $\mathcal{R}$ of a given density operator $\hat{\rho}$ has the lowest possible norm $\|\mathcal{R}\|_{D}=\sqrt{\langle\mathcal{R}, \mathcal{R}\rangle_{D}}$ among all the possible quasiprobability representations of $\hat{\rho}$. We call the space $Z$ the 'zero representation space', since it contains all the quasiprobabilities which correspond to $\hat{\rho}=0$. 
Note that we can extend the definition of the mapping $C_{o}$ from the real space $O_{+}$ of Hermitian operators to the complex space $O=O_{+} \oplus i O_{+}$of non-Hermitian operators. Indeed, an arbitrary operator $\hat{o}$ can be decomposed into Hermitian $\hat{o}^{+}$and skew-Hermitan $\hat{o}^{-}$parts:

$$
\hat{o}=\hat{o}^{+}+\hat{o}^{-} .
$$

However, $i \hat{o}^{-}$is a hermitian operator. Therefore, we can map $\hat{o}$ as:

$$
C_{o} \hat{o}=C_{o}\left\{\hat{o}^{+}+\hat{o}^{-}\right\}=C_{o} \hat{o}^{+}-i C_{o}\left\{i \hat{o}^{-}\right\} .
$$

Now, if we extend the real space $S_{+}$to the complex space $S=S_{+} \oplus i S_{+}$, the mappig $C_{o}$ becomes a bijection between $O$ and $S$. For example, if the stochastic representation is sufficiently well-defined, we can map the nondiagonal projections $|n\rangle\langle m|$ of some orthonormal basis $\{|n\rangle\}$, onto

$$
C_{o}\{|n\rangle\langle m|\}=\mathcal{O}_{n m}(\lambda) .
$$

Next, we should determine whether the trace formula (11) is valid for non-hermitian $\hat{o}$ ? This is indeed the case: since the relation (11) is linear with respect to $\hat{o}$ and with respect to $\mathcal{O}(\lambda)$, we can split $\hat{o}$ into hermitian and skew-hermitian parts, and perform the same operation as in Eq. (15).

\subsection{Expansion over overcomplete basis}

One important consequence of the arguments in the preceding section is that, using the map $C_{o}$, we can define the inverse mapping for the density operators:

$$
\langle m|\hat{\rho}| n\rangle=\operatorname{Tr}(|n\rangle\langle m|) \hat{\rho}=\int d \lambda \mathcal{O}_{n m}(\lambda) \mathcal{P}(\lambda) .
$$

Introducing the operator $\hat{\Lambda}(\lambda)$ defined by its matrix elements:

$$
\langle m|\hat{\Lambda}(\lambda)| n\rangle=C_{o}\{|n\rangle\langle m|\}=\mathcal{O}_{n m}(\lambda),
$$

we obtain the expansion of density operators over the operator basis $\hat{\Lambda}(\lambda)$ :

$$
\hat{\rho}=\int d \lambda \hat{\Lambda}(\lambda) \mathcal{P}(\lambda)
$$

This equation defines the mapping from the space of admissible quasiprobability distributions $D$ to a certain space $P$ of operators, which contains the convex set of physical density operators $P_{+}: P_{+} \subset P$. Note that since the matrix elements of $\hat{\Lambda}(\lambda)$ belong to the space $S$, the operator basis $\hat{\Lambda}(\lambda)$ is overcomplete (if the space $\mathcal{Z}$ is non-trivial):

$$
\int d \lambda \hat{\Lambda}(\lambda) \mathcal{Z}(\lambda)=0 \text { for any } \mathcal{Z} \in Z
$$

Now, by substitution of the density operator expansion (19) into the trace formula (11), we obtain the trace representation of the map $C_{o}$ :

$$
C_{o} \hat{o}=\mathcal{O}(\lambda)=\operatorname{Tr} \hat{o} \hat{\Lambda}(\lambda) .
$$

Since every operator can be expanded over the projections $|n\rangle\langle m|$, and $C_{o}$ is a bijection, we conclude that $\mathcal{O}_{n m}(\lambda)$ is a basis in the space $S$. 


\subsection{The mapping for density operators $C_{\rho}$}

In this section, we will connect the properties of the mapping $C_{\rho}$ with those of $C_{o}$. Let us denote by $C_{\rho}^{(R)}$ the inverse of the mapping defined by the expansion $(19), \mathcal{R}=C_{\rho}^{(R)} \hat{\rho}$, and $C_{\rho}^{(R)}: P \rightarrow \mathcal{R}$ is a bijection. Then, the physical and mathematical consistency of our model requires that $C_{\rho} \hat{\rho}-C_{\rho}^{(R)} \hat{\rho} \in Z$ for any $\hat{\rho}$. This means that we have a (non)linear operator $C_{\rho}-C_{\rho}^{(R)}$ which for every density operator $\hat{\rho}$, associates a certain quasiprobability $\mathcal{Z} \in Z$. As long as the density operators $\hat{\rho}$ and the quasiprobabilities $\mathcal{R}(\lambda)$ are in a one-to-one correspondence, we may write:

$$
C_{\rho} \hat{\rho}=\mathcal{R}(\lambda)+\alpha[\mathcal{R}](\lambda)
$$

where $\mathcal{R}=C_{\rho}^{(R)} \hat{\rho}$, and $\alpha$ is a certain (non)linear mapping, $\alpha: R \rightarrow Z$. Therefore, the existence of the mapping $C_{\rho}$ guarantees that we can choose at least one such mapping $\alpha$, that the quasiprobabilities in Eq. (22) become real and positive.

\subsection{The mapping for the Liouville superoperator $C_{s}$}

Consider the action of the quantum Liouville superoperator:

$$
\hat{\rho}^{\prime}=[\widehat{H}, \hat{\rho}] / i \hbar
$$

which is a linear operator $P \rightarrow P$ (assuming that $\hat{\rho}^{\prime} \in P$ ). Substituting $\hat{\rho}^{\prime}=\left(C_{\rho}^{(R)}\right)^{-1} \mathcal{R}^{\prime}$ and $\hat{\rho}=\left(C_{\rho}^{(R)}\right)^{-1} \mathcal{R}$ into Eq. (23), we obtain the operator $\mathcal{H}^{(R)}: R \rightarrow R$ defined as:

$$
\mathcal{R}^{\prime}(\lambda)=\mathcal{H}^{(R)}[\mathcal{R}](\lambda)=\frac{1}{i \hbar} C_{\rho}^{(R)}\left\{\int d \lambda^{\prime}\left[\widehat{H}, \hat{\Lambda}\left(\lambda^{\prime}\right)\right] \mathcal{R}\left(\lambda^{\prime}\right)\right\}(\lambda) .
$$

Here, we assume that the space $D$ of admissible quasiprobabilities is such that we can move the linear operators through the integration whenever necessary. In this manner, we obtain the stochastic representation for the Liouville superoperator. Note that the definition of $\mathcal{H}^{(R)}$ [Eq. (24)] can be extended to the whole space $D$ in a consistent manner: we have $\mathcal{H}^{(R)}[\mathcal{R}+\mathcal{Z}]=\mathcal{H}^{(R)}[\mathcal{R}]$ for any quasiprobability $\mathcal{Z} \in Z$ due to the basis overcompleteness (20). Now, suppose that there exists another linear operator $\mathcal{H}: D \rightarrow D$, which differs from $\mathcal{H}^{(R)}$, but which also represents the superoperator (23). Then, the physical and mathematical consistency of our model requires that $\left(\mathcal{H}-\mathcal{H}^{(R)}\right) \mathcal{P} \in Z$ for any $\mathcal{P} \in D$. Introducing the space $A$ of linear operators $\alpha: D \rightarrow Z$, we obtain the general form of all possible representations of the superoperator (23):

$$
\mathcal{P}^{\prime}(\lambda)=\mathcal{H}[\mathcal{P}](\lambda)=\mathcal{H}^{(R)}[\mathcal{P}](\lambda)+\alpha[\mathcal{P}](\lambda)
$$

for arbitrary $\alpha \in A$. The existence of the mapping $C_{s}$, which is postulated in Eq. (7), guarantees that we can always choose such $\alpha \in A$ that $\mathcal{H}$ becomes a generator of a stochastic process.

We see that the mapping $C_{o}$ [Eq. (5)] together with the trace formula (6) are the most important components of the stochastic representation: they completely define the mathematical structure of our stochastic representation, which is given by Eqs. (19), (21), (22) and (25). 


\subsection{Quasiprobability master equation}

Having described the general form for all the ingredients in our stochastic representation, let us now discuss under what conditions we can obtain the master equation (8), and when it represents indeed the exact quantum dynamics given by the quantum Liouville equation (3). We have discussed the representation of the right hand side of Eq. (3) in the previous section, and there, we have assumed that the space $D$ is selected so that such a representation is valid. Then, the general form of the master equation is:

$$
\frac{\partial}{\partial t} \mathcal{P}=\mathcal{H} \mathcal{P}+\alpha[\mathcal{P}]
$$

for arbitrary $\alpha \in A$. The necessary condition for Eq. (26) to be valid for a given initial condition $\mathcal{P}(\lambda, t=0) \in D$ is that the $\alpha$ should be such that at any later time the solution $\mathcal{P}(\lambda, t)$ should belong to the space $D$. Further restrictions come from the left-hand side of Eq. (26): to arrive at the term $\partial \mathcal{P} / \partial t$, we differentiate by $t$ the expansion (19), and we need to move the time derivative through the (usually) improper integral. Therefore, an additional constraint is imposed on the solution of Eq. (26): for each time moment $t$, there should exist an arbirtrary small but finite interval $[t-\epsilon, t+\epsilon]$ such that the improper integral in the expansion (19) is converging uniformly with respect to $t \in[t-\epsilon, t+\epsilon]$. If these conditions are violated, then the results of Monte Carlo simulations would show systematic errors with respect to the exact quantum dynamics Eq. (3), and phenomena like the so-called "spiking behavior" and "unaccounted boundary terms" would be observed [11, 23, 33].

\subsection{Annihilators of the overcomplete basis}

In the previous section, we have seen that the space $A$ of operators $\alpha: D \rightarrow Z$ plays an important role in the stochastic representation. First, these operators $\alpha$ allow us to transform the representation $\mathcal{H}$ [Eq. (25)] of the quantum Liouville superoperator into the form which admits the stochastic interpretation; second, the operators $\alpha$ allow us to adjust the quasiprobability master equation [Eq. (26)] so that it is valid and that it possesses no systematic errors with respect to the exact quantum dynamics [Eq. (3)].

Here, we obtain the characterization of the space $A$ which is more useful in practice [21]. Suppose that the space of admissible quasiprobability distributions $D$ is such that for the operators $\alpha \in A$ there exist their adjoints with respect to the dot product $\langle,\rangle_{S}$ :

$$
\left\langle\mathcal{O}^{*}, \alpha \mathcal{P}\right\rangle_{S}=\left\langle\left(\alpha^{T} \mathcal{O}\right)^{*}, \mathcal{P}\right\rangle_{S} .
$$

Here, by superscript $T$ we have denoted the transposition operation, which is defined as the composition of Hermitian conjugation and the complex conjugation, $\alpha^{T}=\left(\alpha^{\dagger}\right)^{*}$; the domains are $\alpha^{T}: S \rightarrow S$; here $\mathcal{O} \in S$ and $\mathcal{P} \in D$. As far as $\alpha \mathcal{P} \in Z$, we have: $\left\langle\left(\alpha^{T} \mathcal{O}\right)^{*}, \mathcal{P}\right\rangle_{S}=0$ for any $\mathcal{P} \in D$. Therefore, $\alpha^{T} \mathcal{O}$ is an observable which is zero for every state, and we conclude that $\alpha^{T} \mathcal{O}=0$. In particular, since $\langle m|\hat{\Lambda}(\lambda)| n\rangle \in S$, we have:

$$
\alpha^{T} \hat{\Lambda}=0 \text { for any } \alpha \in A .
$$

According to mathematical convention, we call the operators $\alpha^{T}$ "the annihilators of the basis $\hat{\Lambda}(\lambda)^{\prime \prime}$.

The definition (28) has practical benefits due to the way in which one usually constructs the stochastic representations $[12,17,20,22,34]$. Suppose that we are given a concrete physical system. Then, at the first stage, one usually starts from an appropriate self-consistent field model, which takes into account all the major features of this system on a qualitative level. As a rule, this corresponds to the assumption that the density operator 
$\hat{\rho}$ can be well approximated by a certain operator ansatz $\hat{\Lambda}(\lambda)$. At the second stage, one is looking for a way to incorporate the noise into this self-consistent field model, so that the resulting stochastic representation is equivalent to the exact quantum dynamics. Therefore, the common situation is that we are given a certain operator ansatz $\hat{\Lambda}(\lambda)$, and we need to investigate the possibilities that it provides for the stochastic representations. Then, it is much easier to find the differential identities of the form (28), to transpose them, and to add them into the master equation (26), than to find the spaces $Z, D$, and $A$.

\section{Example of a stochastic representation: the stochastic wave-function method}

In our recent work [21], we have studied the stochastic wave-function method of Carusotto et al. [22]. In this method, one selects the $N$-particle Hartree-Fock state projections:

$$
\widehat{\Lambda}\left(\phi, \phi^{+*}\right)=|N: \phi\rangle\left\langle N: \phi^{+}\right|,
$$

as the operator ansatz for the density operator $\hat{\rho}$. Here, the Fock state is defined as $|N: \phi\rangle=\left[\sum_{k} \phi_{k} \widehat{a}_{k}^{\dagger}\right]^{N}|0\rangle / \sqrt{N !}$. Therefore, the space $L$ consists of all the vectors $\lambda=$ $\left(\phi, \phi^{*}, \phi^{+}, \phi^{+*}\right)$. Now, in order to define the stochastic representation, we need to find all the necessary mappings for operators and superoperators.

\subsection{Operator correspondences for the density operator}

In [21], the mapping $C_{\rho}$ is constructed in the following way. Suppose that we have presented the density operator $\hat{\rho}$ as an antinormally ordered operator series,

$$
\hat{\rho}=c_{0}+\sum_{m=1}^{N} \prod_{j=1}^{m}\left\{\sum_{k_{j}=1}^{\mathcal{N}} \widehat{a}_{k_{j}}\right\} \prod_{j=1}^{m}\left\{\sum_{k_{j}^{\prime}=1}^{\mathcal{N}} \widehat{a}_{k_{j}^{\prime}}^{\dagger}\right\} h\left(\mathbf{x}_{k_{1}}, \ldots, \mathbf{x}_{k_{m}} \mid \mathbf{x}_{k_{1}^{\prime}}, \ldots, \mathbf{x}_{k_{m}^{\prime}}\right),
$$

where $\mathcal{N}$ is the size of the discretization lattice. Then, the corresponding quasiprobability $\mathcal{P}(\lambda)=C_{\rho} \hat{\rho}$ is found within two steps. First, we perform the replacements $\widehat{a}_{k_{j}} \rightarrow \phi_{k_{j}}^{*}$, $\widehat{a}_{k_{j}^{\prime}}^{\dagger} \rightarrow \phi_{k_{j}^{\prime}}$ in Eq. (30), and multiplicate each monomial by a normalization factor:

$$
\begin{aligned}
\mathcal{P}(\lambda)= & \left\{\frac{1}{\Gamma_{\mathcal{N}}(N) N !} c_{0}+\frac{1}{\Gamma(m+N) N !} \sum_{m=1}^{\infty} \prod_{j=1}^{m}\left\{\sum_{k_{j}=1}^{\mathcal{N}} \phi_{k_{j}}^{*}\right\} \prod_{j=1}^{m}\left\{\sum_{k_{j}^{\prime}=1}^{\mathcal{N}} \phi_{k_{j}^{\prime}}\right\}\right. \\
& \left.\times h\left(\mathbf{x}_{k_{1}}, \ldots, \mathbf{x}_{k_{m}} \mid \mathbf{x}_{k_{1}^{\prime}}, \ldots, \mathbf{x}_{k_{m}^{\prime}}\right)\right\} \boldsymbol{\delta}_{S}(\|\boldsymbol{\phi}\|-1) \boldsymbol{\delta}\left(\boldsymbol{\phi}^{+}-\boldsymbol{\phi}\right),
\end{aligned}
$$

where the normalization factor is

$$
\Gamma_{\mathcal{N}}(p)=S_{2 \mathcal{N}-1} \frac{(\mathcal{N}-1) !}{(\mathcal{N}-1+p) !}
$$

and $S_{2 \mathcal{N}-1}$ is the volume of the unit sphere in $\mathbb{C}^{\mathcal{N}}$. However, the quasiprobability $\mathcal{P}(\lambda)$ which is obtained in Eq. (31), is not positive in general. We make it positive within the second step, by employing the method of Carusotto et al. [22, 35]; we write the density operator expansion for the basis (29),

$$
\hat{\rho}=\int \prod_{k=1}^{\mathcal{N}} d \phi_{k} d \phi_{k}^{*} \prod_{k=1}^{\mathcal{N}} d \phi_{k}^{+} d \phi_{k}^{+*} \mathcal{P}\left(\boldsymbol{\phi}, \boldsymbol{\phi}^{*}, \boldsymbol{\phi}^{+}, \boldsymbol{\phi}^{+*}\right)|N: \boldsymbol{\phi}\rangle\left\langle N: \boldsymbol{\phi}^{+}\right| .
$$


Then we separate the phase of $\mathcal{P}(\lambda)$ :

$$
\mathcal{P}(\lambda)=|\mathcal{P}(\lambda)| e^{i \xi(\lambda)} .
$$

Next, we absorb the phase into $|N: \phi\rangle\left\langle N: \phi^{+}\right|$:

$$
\hat{\rho}=\int \prod_{k=1}^{\mathcal{N}} d \phi_{k} d \phi_{k}^{*} \prod_{k=1}^{\mathcal{N}} d \phi_{k}^{+} d \phi_{k}^{+*}|\mathcal{P}(\lambda)|\left|N: \phi e^{i \xi(\lambda) / 2 N}\right\rangle\left\langle N: \phi^{+} e^{-i \xi(\lambda) / 2 N}\right| .
$$

If we change the variables as $\phi e^{i \xi(\lambda) / 2 N} \rightarrow \boldsymbol{\phi}, \boldsymbol{\phi}^{+} e^{-i \xi(\lambda) / 2 N} \rightarrow \boldsymbol{\phi}^{+}$, and calculate the Jacobian determinant, we can return to form $(33)$, where $\mathcal{P}$ is now real and positive. However from the point of view of the stochastic simulations, one may stop at (35); we sample the initial conditions with the probability distribution $|\mathcal{P}(\lambda)|$, and each time we multiply the sampled wave functions by the phase factor $e^{ \pm i \xi(\lambda) / 2 N}$.

\subsection{Operator correspondences for the observables}

The mapping $C_{o}$ is constructed in the following way. Suppose that we have presented the observable operator $\hat{O}$ as a normally ordered operator series:

$$
\hat{O}=c_{0}+\sum_{m=1}^{N} \prod_{j=1}^{m}\left\{\sum_{k_{j}=1}^{\mathcal{N}} \widehat{a}_{k_{j}}^{\dagger}\right\} \prod_{j=1}^{m}\left\{\sum_{k_{j}^{\prime}=1}^{\mathcal{N}} \widehat{a}_{k_{j}^{\prime}}\right\} h\left(\mathbf{x}_{k_{1}}, \ldots, \mathbf{x}_{k_{m}} \mid \mathbf{x}_{k_{1}^{\prime}}, \ldots, \mathbf{x}_{k_{m}^{\prime}}\right) .
$$

Due to the linearity of the mapping $C_{o}$, it is sufficient to consider the terms

$$
\hat{O}=\widehat{a}_{k_{1}}^{\dagger} \ldots \widehat{a}_{k_{l}}^{\dagger} \widehat{a}_{k_{1}^{\prime}} \ldots \widehat{a}_{k_{m}^{\prime}}
$$

Then, we employ the trace representation (21):

$$
\begin{aligned}
C_{o} \widehat{a}_{k_{1}}^{\dagger} \ldots \widehat{a}_{k_{l}}^{\dagger} \widehat{a}_{k_{1}^{\prime}} \ldots \widehat{a}_{k_{m}^{\prime}}=\operatorname{Tr} \widehat{a}_{k_{1}}^{\dagger} \ldots \widehat{a}_{k_{l}}^{\dagger} \widehat{a}_{k_{1}^{\prime}} \ldots \widehat{a}_{k_{m}^{\prime}}|N: \phi\rangle\left\langle N: \phi^{+}\right| \\
\\
=\phi_{k_{1}}^{+*} \ldots \phi_{k_{l}}^{+*} \phi_{k_{1}^{\prime}} \ldots \phi_{k_{m}^{\prime}} \delta_{l m} \frac{N !^{2}}{(N-m) !}\left\langle\phi^{+} \mid \phi\right\rangle^{N-m} .
\end{aligned}
$$

\subsection{Operator correspondences of the star-product type}

In order to find the mapping $C_{s}$ for the quantum Liouville superoperator, we proceed in a constructive way [21]. We note that for a Hamiltonian of the form (1), the action of the Liouville superoperator $[\widehat{H}, \cdot] / i \hbar$ is constructed from the multiplications by $\widehat{a}_{s}$ and $\widehat{a}_{s}^{\dagger}$ from the left and from the right. Since such multiplications, e. g., $\widehat{a}_{s} \times \cdot$ and $\cdot \times \widehat{a}_{s}$, are also superoperators, we find the mapping $C_{s}$ for the left multiplication by a number-conserving normally-ordered product of creation and annihilation operators [21]:

$$
C_{s}\left[\widehat{a}_{k_{1}}^{\dagger} \ldots \widehat{a}_{k_{l}}^{\dagger} \widehat{a}_{k_{1}^{\prime}} \ldots \widehat{a}_{k_{l}^{\prime}} \times \cdot\right]=(-1)^{l} \frac{\partial}{\partial \phi_{k_{1}}} \ldots \frac{\partial}{\partial \phi_{k_{l}}} \phi_{k_{1}^{\prime}} \ldots \phi_{k_{l}^{\prime}},
$$

and for the right multiplication,

$$
C_{s}\left[\cdot \times \widehat{a}_{k_{1}}^{\dagger} \ldots \widehat{a}_{k_{l}}^{\dagger} \widehat{a}_{k_{1}^{\prime}} \ldots \widehat{a}_{k_{l}^{\prime}}\right]=(-1)^{l} \frac{\partial}{\partial \phi_{k_{1}^{\prime}}^{+*}} \ldots \frac{\partial}{\partial \phi_{k_{l}^{\prime}}^{+*}} \phi_{k_{1}}^{+*} \ldots \phi_{k_{l}}^{+*} .
$$


Using these relations, we can evaluate the operator $\mathcal{H}$ :

$$
\begin{aligned}
\mathcal{H}=C_{s}[\widehat{H}, \cdot] / i \hbar=-\sum_{r} & \frac{\partial}{\partial \phi_{r}} \mathcal{A}_{r}[\phi]-\sum_{r} \frac{\partial}{\partial \phi_{r}^{+*}}\left(\mathcal{A}_{r}\left[\phi^{+}\right]\right)^{*} \\
& +\frac{1}{2} \sum_{r, s} \frac{\partial}{\partial \phi_{r}} \frac{\partial}{\partial \phi_{s}} V_{r s} \frac{\phi_{r} \phi_{s}}{i \hbar}+\frac{1}{2} \sum_{r, s} \frac{\partial}{\partial \phi_{r}^{+*}} \frac{\partial}{\partial \phi_{s}^{+*}}\left(V_{r s} \frac{\phi_{r}^{+} \phi_{s}^{+}}{i \hbar}\right)^{*} .
\end{aligned}
$$

We see that the operator $\mathcal{H}$ does not have the required form of the genuine Fokker-Planck operator in complex variables [21]. In particular, the drift terms lack their complex conjugates, and the diffusion matrix is not Hermitian. Nevertheless, we can solve this issue by adding the annihilator $\alpha$ to the master equation (41).

\subsection{Annihilators of the overcomplete basis}

As was described in Sec. 3.6, the properties of the stochastic representation are most conveniently described in terms of the differential identities of form (28). In work [21], we find the following differential identities for the Hartree-Fock state projections (29): (i) the analyticity of the basis $\widehat{\Lambda}\left(\phi, \phi^{+*}\right)$ with respect to its variables;

$$
\frac{\partial}{\partial \phi_{k}^{*}} \widehat{\Lambda}\left(\phi, \phi^{+*}\right)=0, \frac{\partial}{\partial \phi_{k}^{+}} \widehat{\Lambda}\left(\phi, \phi^{+*}\right)=0
$$

(ii) the homogeneity of the Hartree-Fock state,

$$
\begin{gathered}
{\left[N-\sum_{k} \phi_{k} \frac{\partial}{\partial \phi_{k}}\right] \hat{\Lambda}\left(\boldsymbol{\phi}, \boldsymbol{\phi}^{+*}\right)=0,} \\
{\left[N-\sum_{k} \phi_{k}^{+*} \frac{\partial}{\partial \phi_{k}^{+*}}\right] \hat{\Lambda}\left(\boldsymbol{\phi}, \boldsymbol{\phi}^{+*}\right)=0 .}
\end{gathered}
$$

From expressions (42)-(44), we conclude that the operator $\alpha$, in the general form (26) of the master equation, has the form:

$$
\alpha=\sum_{k} \frac{\partial}{\partial \phi_{k}^{*}} l_{k}^{*}+\sum_{k} \frac{\partial}{\partial \phi_{k}^{+}} l_{k}^{+}+\left[N+\sum_{k} \frac{\partial}{\partial \phi_{k}} \phi_{k}\right] d+\left[N+\sum_{k} \frac{\partial}{\partial \phi_{k}^{+*}} \phi_{k}^{+*}\right] d^{+*} .
$$

Here, $l_{k}, l_{k}^{+}, d, d^{+}$are some linear operators $D \rightarrow D$.

In work [21] it is discussed in detail how we can choose such parameters $l_{k}, l_{k}^{+}, d, d^{+}$ that the master equation (26) with $\mathcal{H}$ [Eq. (41)] becomes the Fokker-Planck equation, and satisfies the validity conditions discussed in Sec. 3.5.

\section{Conclusion}

In this work we have considered the most general definition of the classical stochastic representation of the many-body quantum dynamics. Such representation should provide several mappings: for density operators $C_{\rho}$, for observables $C_{o}$, and for superoperators $C_{s}$. The most important postulated property of the stochastic representation is the classical representation of the trace formula for average values of the observables.

The main result of this work is that when the mathematical properties of the stochastic representation are regular enough, and it is physically consistent, then the stochastic representation is reduced to the expansion of the density operator over a certain overcomplete operator basis $\hat{\Lambda}(\lambda)$. This operator basis $\hat{\Lambda}(\lambda)$ defines all the propertes of the stochastic representation: the general form of all the mappings. Moreover, the overcompleteness of the 
basis leads to the existence of equivalent transformations of quasiprobability master equations, which allows us to perform their stochastic unraveling. Such a structure is already known to occur in the representations of quantum mechanics in generalized phase spaces [23-26]. We also demonstrate that the stochastic wave-function methods [8, 12-20, 36] also fall into this category of stochastic representations.

\section{Acknowledgements}

This work is supported by the Russian Foundation for Basic Reseach (grant 14-0231109).

\section{References}

[1] Aksenova E. V., Kuz'min V. L., Romanov V. P. Coherent backscattering of light in nematic liquid crystals. J. Exp. Theor. Phys., 2009, 108, P. 516.

[2] Aksenova E. V., Kokorin D. I., Romanov V. P. Simulation of coherent backscattering of light in nematic liquid crystals. J. Exp. Theor. Phys., 2012, 115, P. 337.

[3] Aksenova E. V., Kokorin D. I., Romanov V. P. Simulation of radiation transfer and coherent backscattering in nematic liquid crystals. Phys. Rev. E., 2014, 89, P. 052506.

[4] Drummond P. D., Gardiner C. W. Generalized p-representations in quantum optics. J. Phys. A: Math. Gen., 1980, 13, P. 2353.

[5] Deuar P., Drummond P. D. First-principles quantum dynamics in interacting bose gases: I. the positive p representation. J. Phys. A: Math. Gen., 2006, 39, P. 1163.

[6] Savage C. M., Schwenn P. E., Kheruntsyan K. V. First-principles quantum simulation of dissociation of molecular condensates: Atom correlations in momentum space. Phys. Rev. A., 2006, 74, P. 033620.

[7] Deuar P., Drummond P. D. Correlations in a bec collision: First-principles quantum dynamics with 150 000 atoms. Phys. Rev. Lett., 2007, 98, P. 120402.

[8] Juillet O., Gulminelli F. Exact pairing correlations for one-dimensionally trapped fermions with stochastic mean-field wave functions. Phys. Rev. Lett., 2004, 92, P. 160401.

[9] Biele R., D'Agosta R. A stochastic approach to open quantum systems. J. Phys.: Condens. Matter., 2012, 24, P. 273201.

[10] Lee M. D., Ruostekoski J. Classical stochastic measurement trajectories: Bosonic atomic gases in an optical cavity and quantum measurement backaction. Phys. Rev. A., 2014, 90, P. 023628.

[11] Gardiner C. W., Zoller P. Quantum Noise: a Handbook of Markovian and Non-Markovian Quantum Stochastic Methods with Applications to Quantum Optics. Springer Series in Synergetics. Berlin, Germany, Springer, 2004. ISBN: 3540223010, 9783540223016.

[12] Carusotto I., Castin Y. An exact reformulation of the bose-hubbard model in terms of a stochastic gutzwiller ansatz. New. J. Phys., 2003, 5, P. 91.

[13] Wilkie J. Variational principle for stochastic wave and density equations. Phys. Rev. E., 2003, 67, P. 017102.

[14] Tessieri L., Wilkie J., etinba M. Exact norm-preserving stochastic time-dependent hartree-fock. J. Phys. A: Math. Gen., 2005, 38, P. 943.

[15] Montina A., Castin Y. Exact bcs stochastic schemes for a time-dependent many-body fermionic system. Phys. Rev. A., 73, P. 013618.

[16] Wilkie J., Wong Y. M., Ng R. Survey of exact n-body decompositions of stochastic scalar-jastrow-hartree form. Chem. Phys., 2010, 369, P. 43.

[17] Lacroix D. Stochastic schroedinger equation from optimal observable evolution. Ann. Phys., 2007, 322, P. 2055.

[18] Lacroix D. Optimizing stochastic trajectories in exact quantum-jump approaches of interacting systems. Phys. Rev. A., 2005, 72, P. 013805.

[19] Breuer H.-P. Exact quantum jump approach to open systems in bosonic and spin baths. Phys. Rev. A., 2004, 69, P. 022115.

[20] Lacroix D., Ayik S. Stochastic quantum dynamics beyond mean field. Eur. Phys. J. A., 2014, 50, P. 95.

[21] Polyakov E. A., Vorontsov-Velyaminov P. N. Quasiprobability distributions in stochastic wave-function methods. Phys. Rev. A., 2015, 91, P. 042107. 
[22] Carusotto I., Castin Y., Dalibard J. N-boson time-dependent problem: A reformulation with stochastic wave functions. Phys. Rev. A., 2001, 63, P. 023606.

[23] Deuar P., Drummond P. D. Gauge p representations for quantum-dynamical problems: Removal of boundary terms. Phys. Rev. A., 2002, 66, P. 033812.

[24] Corney J. F., Drummond P. D. Gaussian quantum operator representation for bosons. Phys. Rev. A., 2003, 68, P. 063822.

[25] Corney J. F., Drummond P. D. Gaussian phase-space representations for fermions. Phys. Rev. B., 2006, 73, P. 125112.

[26] Quantum dynamics in ultracold atomic physics / Qiong-Yi He, Margaret D. Reid, Bogdan Opanchuk et al. Front. Phys., 2012, 7, P. 16.

[27] Błaszak M., Domański Z. Phase space quantum mechanics. Ann. Phys. (N. Y.), 2012, 327, P. 167-211.

[28] Gardiner C. Stochastic Methods: A Handbook for the Natural and Social Sciences. Springer Series in Synergetics, Berlin, Germany, Springer, 2009. ISBN: 3540707123, 9783540707127.

[29] Frenkel D., Smit B. Understanding Molecular Simulation. New York : Academic Press, 2001. ISBN: $0122673514,9780122673511$.

[30] Cahill K. E., Glauber R. J. Ordered expansions in boson amplitude operators. Phys. Rev., 1969, 177, P. 1857.

[31] Cahill K. E., Glauber R. J. Density operators and quasiprobability distributions. Phys. Rev., 1969, 177, P. 1882.

[32] Cahill K. E., Glauber R. J. Density operators for fermions. 1999, 59, P. 1538.

[33] Gilchrist A., Gardiner C. W., Drummond P. D. Positive p representation: Application and validity. Phys. Rev. A., 1997, 55, P. 3014.

[34] Juillet O., Chomaz P. Exact stochastic mean-field approach to the fermionic many-body problem. Phys. Rev. Lett., 2002, 88, P. 142503.

[35] Carusotto I., Castin Y. Exact reformulation of the bosonic many-body problem in terms of stochastic wave functions: an elementary derivation. Ann. Henri Poincaré, 2003, 4, P. S783.

[36] Breuer H.-P. The non-markovian quantum behaviour of open systems: An exact monte carlo method employing stochastic product states. Eur. Phys. J. D., 2004, 29, P. 105. 